\title{
Köpekte Beynin 3 Tesla Manyetik Rezonans ile 3 Boyutlu Multiplanar
}

\section{Rekonstruksiyonu}

\author{
Çă̆daş OTO \\ Ankara Üniversitesi, Veteriner Fakültesi, Anatomi Anabilim Dalı, Ankara, Türkiye.
}

Geliş Tarihi: 13.01.2017

Kabul Tarihi: 06.02.2017

\begin{abstract}
Özet: Bu çalışmanın amacı köpek beyninin 3 boyutlu anatomisinin 3 tesla manyetik rezonans tarama görüntülerinin multiplanar rekonstruksiyonu aracılığıyla in situ olarak gösterilmesidir. Neurocranial yapıların anatomik detaylarının gösterilmesi için 9 adet yetişkin mesaticephalic ırk köpek kullanıldı. Bu amaçla T1 ve T2-ağırıılı space sekans taramalarının üç boyutlu yeniden şekillendirilmiş görüntülerinden yararlanıldı. 3D-IR-MPR sekansı ile alınan görüntüler, baş ve beyine ait derin yapıların ayrıntılı olarak görüntülenmesini sağladı.
\end{abstract}

Anahtar Kelimeler: Anatomi, Beyin, Köpek, Manyetik Rezonans görüntüleme, Multiplanar rekonstruksiyon.

\section{Dimentional Multiplanar Reconstruction of the Brain at 3 Tesla Magnetic Resonance in Dog}

\begin{abstract}
The purpose of the study was to show three-dimentional anatomy of the dog brain in situ by using multiplanar reconstruction of the magnetic resonance scanning images at 3 tesla. The nine adult mesaticephalic dogs were used for defining the anatomical details of neurocranial structures. The data are obtained from 3D reformatted images derived by T1 and T2-weighted space sequence scans. Three-dimensional inversion recovery multiplanar reconstruction (3D-IR-MPR) sequence provided excellent visualization for the inner structures of the head and brain.
\end{abstract}

Keywords: Anatomy, Brain, dog, Magnetic resonance imaging, Multiplanar reconstruction.

\section{Giriş}

Manyetik rezonans görüntüleme, yumuşak dokular üzerinde yüksek doku kontrastı gösterebilme özelliği sayesinde günümüzde başta beyin olmak üzere merkezi sinir sistemi ve vücuttaki diğer yumuşak dokuların araştırma, tanı ve tedavi amacıyla görüntülenmesinde en yaygın kullanılan tıbbi görüntüleme yöntemlerinden biridir (Broome ve Broome, 2016; Oto ve ark., 2011; Schmitt ve ark., 2004). Non-invaziv olması, bilinen bir zararının olmaması, gittikçe kısalan tarama süreleri ile elde edilen üstün görüntü kalitesi ve multiplanar 3 boyutlu görüntü alınabilmesi sistemin önemli avantajlarıdır (Broome ve Broome, 2016; Brown, 2016; Schmitt ve ark., 2004;).

Tüm dünyada son 10 yılda insanların yanısıra veteriner hekimlik alanında da MR kullanımı artmaktadır (Oto ve ark., 2011; Schmidt ve ark., 2012; Gray-Edwards ve ark., 2014; Fletcher ve Saveraid, 2016). Beyin ve ilişkili yapılar anatomi eğitiminde evcil memeli örneği olarak kullanılan büyük ruminant Schmidt ve ark., (2012); küçük ruminant Ella ve ark., (2016); Ella ve Keller (2015); Schmidt ve ark., (2012); tektırnaklı Arencibia ve ark., (2001); Chaffin ve ark., (1997); Oto ve Hazıroğlu (2011); Oto (2010); Vazquez ve ark., (2001); domuz Fang ve ark., (2005); Oto ve ark., (2011); Rosendal ve ark., (2009); Watanebe ve ark., (2001); kedi GrayEdwards ve ark., (2014); Lola ve ark., (1995) ve köpeklerde Assheuer ve ark., (1997); Byeong ve ark., (2009) ve Fletcher ve Saveraid (2016) tarafından görüntülenmiş ve kesitsel anatomi açısından incelenmiştir. Son yıllarda geliştirilen sekanslar aracılığıyla dokuların normal pozisyonlarındaki üç boyutlu görüntülerinin de $M R$ ile elde edilmesi mümkün olmaktadır (Phal ve ark., 2008; Brown, 2016). Yapılan literatür incelemesinde carnivor örneği olarak modellenen köpeklerde hem anatomi hem de klinik bazlı çok sayıda MR araştırması olmasına karşın beyin ve ilişkili yapıların 3 boyutlu görüntülerini içeren çalışmaların eksik olduğu düşünülmektedir. Bu çalışmanın amacı köpek kafasının MR taraması ile elde edilecek 3 boyutlu görüntüler üzerinden özellikle anatomi eğitiminde, kafatası içerisinde tamamen kapalı olarak konumlanmış ve karmaşık bir yapıya sahip beyin gibi bir organın anatomisinin ve çevresindeki yapılarla ilişkisinin anlaşımasını kolaylaştıracak bir referans oluşturmaktır.

\section{Materyal ve Metot}

Çalışmada Bilkent Üniversitesi Hayvan Deneyleri Etik Kurulu'nun 2016/44 sayılı izni ile herhangi bir merkezi sinir sistemi hastalığı gözlenmeyen, 6 adet yetişkin mesaticephalic iri ırk köpek ile 3 adet Ankara Üniversitesi Veteriner Fakültesi Anatomi Anabilim Dalı'nda kadavra olarak değerlendirilen köpek başı kullanıldı. Çalışma için 
Atropin+Ksilazin+Dormitol kombinasyonu ile premedikasyon ve sedasyonu sağlanan köpekler ve kadavralara ait başlar prone pozisyonda yerleştirildikten sonra, 3 Tesla MR (Trio, Siemens, Erlangen, Germany) ile 32 kanalı insan kafa koili kullanılarak tarandı. Dorsal, sagittal ve transversal düzlemler üzerinde T1-ağırlıklı space ve T2-ağırlıklı space sekansları ile alınan görüntülerin, sistem dahilindeki Leonardo Workstation yazılımı (Siemens Medical Solutions, Erlangen, Germany) vasitasıyla 3 boyutlu rekonstruksiyon yapıldı. Taramalar sırasında T1ağılıklı görüntüler için parametreler; TE: $12 \mathrm{~ms}$; TR: 600 ms; ; Kesit Kalınlığı: 1 mm; FOV: 230×230 mm; Kesit sayısı: 125, Tarama süresi; 8 dakika, T2-ağırlıklı görüntüler için parametreler; TE: 404 ms; TR: 3000 ms; ; Kesit Kalınlığı: 1 mm; FOV: 230×230 mm; Kesit sayısı: 125 Tarama süresi; 11 dakika olarak belirlendi. Görüntüler üzerinde anatomik yapıların belirlenmesi ve isimlendirilmesi anatomi kitapları De lahunta, (1997) ve atlasları Popesko, (1979), MR atlasları Assheuer ve ark., (1997) ve Nomina Anatomica Veterinaria, (2012) temel alınarak yapıldı.

\section{Bulgular}

MR ile üç düzlemde alınan T1 ve T2 ağırlıklı görüntüler üzerinde köpek beyninin kafatası içindeki ölçüleri uzunluk (mid-sagittal düzlemde bulbus olfactorius'dan foramen magnum seviyesine kadar olan mesafe) $96 \pm 1.7 \mathrm{~mm}$; yükseklik (gl.pinealis seviyesinden geçen transversal kesitlerde en geniş iki nokta arası) $51 \pm 0.9 \mathrm{~mm}$; genişlik (lobus piripormis seviyesinden geçen dorsal kesitlerde en geniş iki nokta arası) $62 \pm 1.1 \mathrm{~mm}$ olarak belirlendi. Köpek başlarının T1-ağırlıklı taramaları sonrası rekonstruksiyonu ile elde edilen renklendirilmiş 3 boyutlu görüntülerde (Şekil 1.A) neurocranial yapılara ait anatomik detaylar transversal, sagittal ve dorsal düzlemler üzerinde incelendi ve işaretlendi. Bu görüntülerde beyin dokusunun cihazın sağladığı yüksek doku kontrastı sayesinde diğer çevre dokulardan (kafatası kemikleri, kaslar, yağ dokusu, meninges ve BOS) rahatlıkla ayrılabildiği görüldü (Şekil 1.B, C, D, E, F, G, H, I, J, K, L).

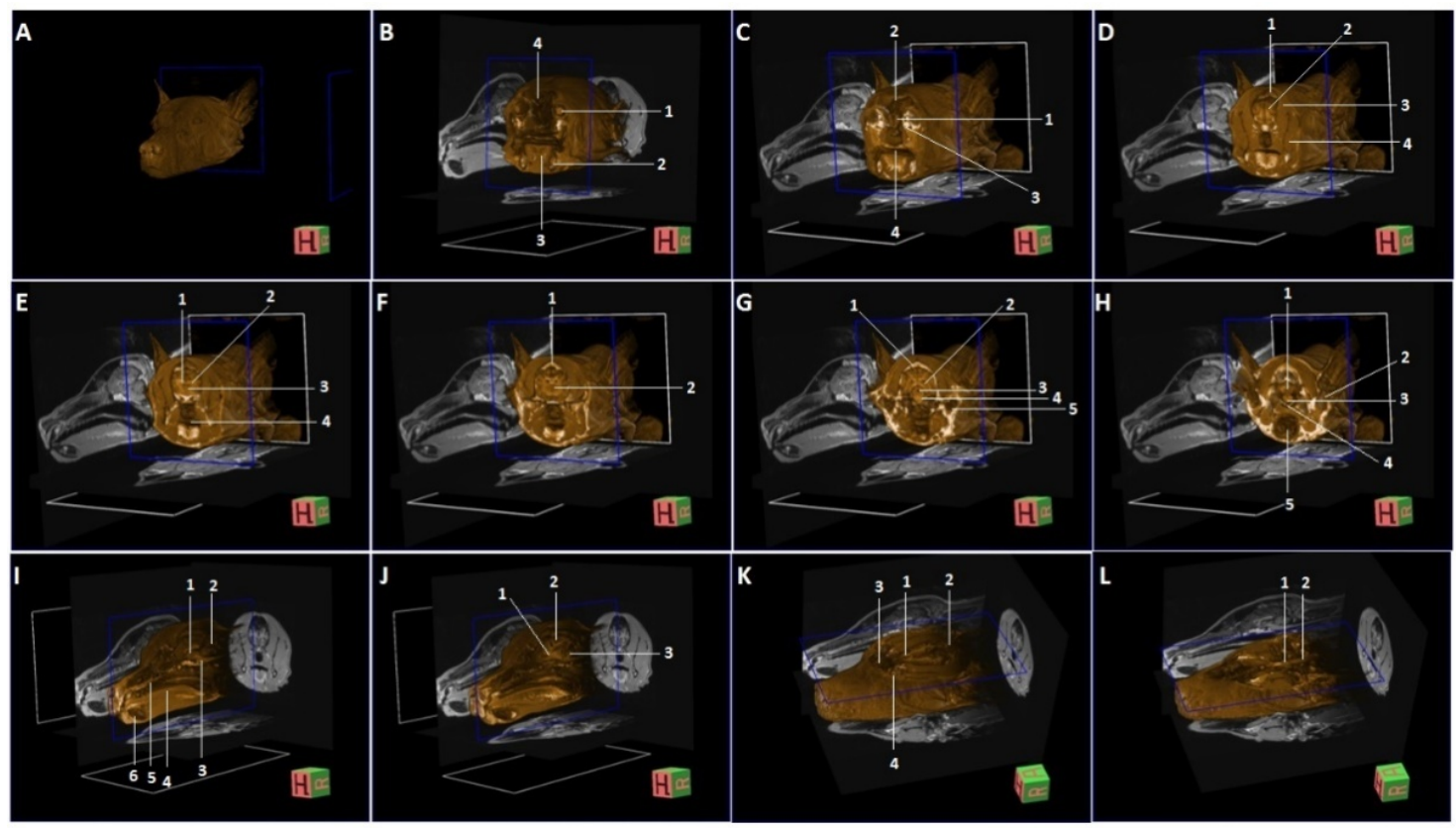

Şekil 1. A-Renklendirilmiş 3B rekonstruksiyon; B-Bulbus oculi seviyesinden geçen transversal görüntü, 1. Lens, 2. Mandibula, 3. Lingua, 4. Sinus frontalis; C- Bulbus olfactorius seviyesinden geçen transversal görüntü, 1.Bulbus olfactorius, 2.Sinus frontalis, 3. Corpus adiposum intraorbitale, 4. Palatum molle; D-Frontal lob seviyesinden geçen transversal görüntü, 1. Os frontale, 2. Substantia grisea, 3. M.temporalis, 4. M.massater; E-Nucleus caudatus seviyesinden geçen transversal görüntü, 1.Corpus callosum, 2. Substantia alba, 3. Nucleus caudatus, 4. Oropharynx; F-Thalamus seviyesinden geçen transversal görüntü, 1. Os parietale, 2. Thalamus; G- Crus cerebri seviyesinden geçen transversal görüntü, 1. Os parietale, 2. Hippocampus, 3. Aqueductus mesencephali, 4. Crus cerebri, 5. Cavum tympani; H-Cerebellum seviyesinden geçen transversal görüntü, 1. Cerebellum, 2. Meatus acusticus externus, 3. Medulla oblongata, 4. Os sphenoidale, 5. Laryngopharynx; I-Mid-sagittal görüntü, 1. Adhesio interthalamica, 2. Cerebellum, 3. Pons, 4. Lingua, 5. Palatum durum, 6. Mandibula; J-Paramedian görüntü, 1.Capsula interna, 2. Sulcus cerebri, 3. Corpus medullare cerebelli; K-Bulbus oculi seviyesinden geçen dorsal görüntü, 1. Tractus olfactorius, 2. Cerebellum, 3. Cavum nasi, 4. Lens; L-GI.pituitaria seviyesinden geçen dorsal görüntü, 1. Gl.pituitaria, 2. Medulla oblongata. 
Benzer olarak beyinde substatia grisea'nın da substantia alba'dan daha hipointens olarak tarandığı ve bu sayede iki bölüm arasında doku görüntü farklılıklarının ayrılabildiği saptandı (Şekil 1.D-2, E-2). Her üç düzlem üzerinde değişik seviyelerden geçen kesitlerde hemispherium cerebri, cerebellum (Şekil 1.H-1, I-2, K-2) ve beyin kökü yanısıra beyin ventrikülleri ve BOS (Şekil 1.G-3) görüntülendi. Buna ek olarak özellikle transversal kesitlerde bulbus olfactorius, nucleus caudatus, thalamus, corpus callosum, tectum mesencephali gibi derin cerebral yapıların anatomik detayları 3 boyutlu görüntüler üzerinde belirlendi ve işaretlendi. Sagittal düzlem üzerindeki kesitler beynin yanında, cavum nasi, palatum, lingua gibi viscerocranium'a ait anatomik yapıların da ayn kesitlerde izlenmesine olanak verdi. Dorsal düzlem kesitlerinde ise beyin ile bulbus oculi'nin görüntülenmesi yapıldı.

Renklendirilmiş görüntülerde kemik korteksi isointens, medullar kısım yoğun hiperintens olarak taranırken kemik doku içindeki hava boşlukları (sinus paranasales) işlenmemiş ham görüntülerden farklı olarak üç boyutlu görüntüleme nedeniyle renksizdi ve boşluk olarak gözlendi (Şekil 1.B-2, B-4). $\mathrm{Bu}$ bağlamda beyni çevreleyen neurocranium yanında maxilla ve mandibula da kolayca çevre dokulardan ayrıldı. Taramalarda genel olarak yağ dokusu hiperintens, kas dokusu hipointens görüntü karakterine sahipti. Özellikle bulbus oculi'nin etrafını çevreleyen intraorbital yağ dokusu parlak beyaz (Şekil 1 C-3), mandibula ve os temporale üzerinde yeralan çiğneme kasları homojen kahverengi tonlarda izlendi (Şekil 1.D-3, D-4).

\section{Tartışma ve Sonuç}

Arencibia ve ark. (2001); Vasquez ve ark. (2001) ve Oto ve ark. (2011) T1-ağırlıklı taramaların anatomik detay açısından T2-ağırlıklı taramalardan daha üstün olduğunu belirtmiş; düzlemler açısından ise transversal düzlem kesitlerinin özellikle derin cerebral yapıların anatomilerinin anlaşılmasında daha yararlı olacağını vurgulamıştır. Yapılan çalışmada T1-ağırlıklı taramaların ve bunlardan rekonstrukte edilen görüntülerin daha fazla anatomik detay verdiği, benzer biçimde yapıların ilişkilendirilmesinde transversal kesitlerin diğer düzlem görüntülerine göre daha anlaşılır olduğu görüldü.

Canlı köpeklerde yapılan yüksek çözünürlüklü taramalar ile karşılaştırıldığında Assheuer ve ark. (1997); Byeong ve ark. (2009) anatomi eğitimi için yapılacak kafa MRG işlemlerinde, ötenazi yapılmış hayvanlara ait görüntülerin de daha önce yapılan çalışmaları destekler biçimde Schmidt ve ark. (2012) tatmin edici anatomik detay sağladığı belirlendi.
Sonuç olarak günümüzde, azalan maliyetlere ve tarama sürelerine bağlı olarak yaygınlaşan kullanımı ve yüksek doku kontrastı sağlayabilmesi gibi avantajları nedeniyle MRG veteriner hekimlik alanında da rutin görüntüleme yöntemleri arasına girmiştir. MRG ile sağlanan ham verilerin rekonstruksiyonu sonucu elde edilen üç boyutlu görüntülerin, hem klinikte baş bölgesine ait özellikle tümör benzeri fiziksel patolojilerin tanısı ve operasyon planlamasının yapılmasında, hem de veteriner anatomi eğitiminde beyin ve ilişkili yapıların daha kolay anlatılması ve anlaşılması açısından önemli bir kaynak olarak kullanılabileceği düşünülmektedir.

\section{Teşekkür}

$\mathrm{Bu}$ çalışmaya katkılarından dolayı UMRAM direktörü Prof. Dr. Ergin ATALAR’a, Atatürk Eğitim ve Araştırma Hastanesi Girişimsel Radyoloji Bölümü öğretim üyesi Doç. Dr. Oktay ALGIN'a ve UMRAM sorumlu radyoloji teknikeri Musa KURNAZ'a teşekkür ederiz.

\section{Kaynaklar}

Arencibia A, Vasquez JM, Ramirez JA, Ramires G, Vilar JM, Rivero MA, Alayon S, Gil F, 2001: Magnetic resonance imaging of normal equine brain. Vet Radiol Ultrasound, 42, 405-408.

Assheuer J, Sager M 1997: MRI and CT Atlas of the Dog. 1st Ed., Blackwell Wissenschafts, Berlin.

Broome M, Broome N, 2016: "MRI". http://www.avmi.net/services/mri, Erişim tarihi; 15.11.2016

Brown G, 2016: "Multiplanar reconstructed magnetic resonance imaging as a tool for anatomical investigation". http://www.ismrm.org/smrt/08/ smrt.doc/, Erişim tarihi; 15.11.2016.

Byeong TK, Ki JK, Dong PJ, Han JY, Lim CY, Park C, Yoo JH, Kim JW, Jung DI, Kim YB, Woo EJ, Cho ZE, Park HM, 2009: Magnetic Resonance Imaging of the Canine Brain at 7t. Vet Radiol Ultrasound, 50, 615-621.

Chaffin MK, Walker MA, McArthur NH, Perris EE, Matthews NS, 1997: Magnetic resonance imaging of the brain of normal neonatal foals. Vet Radiol Ultrasound, 38, 102-111.

De Lahunta A, 1997: Veterinary Neuroanatomy and Clinical Neurology. 3rd Ed., W.B.Saunders Company Philadelphia, London, Toronto, Mexico City, Sydney, Tokyo.

Ella A, Delgadillo JA, Chemineau P, Keller M, 2016: Computation of a High-Resolution MRI 3D Stereotaxic Atlas of the Sheep Brain. J Comp Neurol, 525,3, 676-692.

Ella A, Keller M, 2015: Construction of an MRI 3D high resolution sheep brain template. Magnetic Resonance Imaging, 33, 1329-1337.

Fang $\mathrm{M}$, Li J, Gong X, Antonio G, Lee F, Kwong WH, Wai SM, Yew DT, 2005: Myelination of the pig's brain: A 
correlated MRI and histological study. Neurosignals, 14, 102-108.

Fletcher TF, Saveraid TC, 2016: Canine Brain Mri Atlas. http://vanat.cvm.umn.edu/mMRIBrain/, Erişim tarihi; 15.11 .2016

Gray-Edwards HL, Salibi N, Josephson EM, Hudson JA, Cox NR, Randle AN, McCurdy VJ, Bradbury AM, Wilson DU, Beyers RJ, Denney TS, Martin DR, 2014: High resolution MRI anatomy of the cat brain at 3 Tesla. Journal of Neuroscience Methods, 227, 10-17.

International Committee on Veterinary Gross Anatomical Nomenclature. Nomina Anatomica Veterinaria (N.A.V.), 2012: 6th Ed., Hannover, Columbia, Gent, Sapporo: World association of Veterinary Anatomists.

Hudson, LC, Cauzinille L, Kornegay JN, 1995: Magnetic resonance imaging of the normal feline brain. Vet Radiol Ultrasound, 36, 267-275.

Oto C, Ekim O, Algın O, Şenel OO, İnce N, Hazıroğlu RM, 2011: 3 Tesla Magnetic resonance imaging and multiplanar reconstruction of the brain and its associated structures in pig. Vet J Ankara Univ, 58, 73-78.

Oto C, Hazıroğlu RM, 2011: Magnetic resonance imaging of the guttural pouch (diverticulum tubae auditivae) and its related structures in donkey (Equus asinus). Vet J Ankara Univ, 58, 1-4.

Oto C, 2010: Magnetic resonance imaging of the brain in donkey. Proceeding "The XXVIIth Congress of the European Association of the Veterinary Anatomists, 28-31 July, Paris". Anat Histol Embryol, 39, 311.

Phal PM, Usmanov A, Nesbit GM, Anderson JC, Spencer D, Wang P, Helwig JA, Roberts C, Hamilton BE, 2008:
Qualitative comparison of 3-T and 1,5-T MRI in the evaluation of epilepsy. Neuroradiol, 191, 890-895.

Popesko P, 1979: Atlas of topografical anatomy of the domestic animals. Vol. 1, Head and Neck. 1st Ed., Ferdinand Enke Verlag, Stuttgart.

Rosendal F, Pedersen M, Sangill R, Stødkilde-Jørgensen $H$, Nielsen MS, Bjarkam CR, Sunde N, Sørensen JC, 2009: MRI protocol for in vivo visualization of the Göttingen minipig brain improves targeting in experimental functional neurosurgery. Brain Res Bull, 79, 41-45.

Schmidt MJ, Langen N, Klumpp S, Nasirimanesh F, Shirvanchi P, Ondreka N, Kramer M, 2012: A study of the comparative anatomy of the brain of domestic ruminants using magnetic resonance imaging. The Veterinary Journal, 191, 85-93.

Schmitt F, Grosu D, Mohr C, Purdy D, Salem K, Scott KT, Stoeckel B, 2004: 3 Tesla MRI: successful results with higher field strengths: High field MR. Der Radiologe, 44, 31-48.

Vazquez JM, Rivero M, Gil F. 2001: Magnetic resonance imaging of two normal equine brains and their associated structures. Vet Rec, 148, 229-232.

Watanabe H, Andersen F, Simonsen CZ. 2001: MR-based statistical atlas of the Göttingen minipig brain. Neuroimage, 14, 1089-1096.

Yazışma Adresi: Çağdaş OTO

Ankara Üniversitesi, Veteriner Fakültesi,

Anatomi Anabilim Dalı, Ankara, Türkiye.

e-mail: coto@ankara.edu.tr 